\title{
EFICÁCIA DA FISIOTERAPIA NA MANUTENÇÃO DA CAPACIDADEFUNCIONAL DE IDOSOS PÓS CIRURGIA DE FRATURA PROXIMAL DE FÊMUR
}

\author{
EFFECTIVENESS OF PHYSIOTHERAPY IN MAINTAINING THE FUNCTIONAL \\ CAPACITY OF THE ELDERLY AFTER PROXIMAL FEMUR FRACTURE SURGERY
}

\author{
Alana Freitas Santos ${ }^{1}$ \\ Kauara Vilarinho Santana Vieira ${ }^{2}$
}

RESUMO: O envelhecimento é uma fase da vida influenciada por diversos fatores e determinantes, como a diminuição das funções fisiológicas e das capacidades físicas. A perda de força, massa óssea, flexibilidade, velocidade, redução da massa muscular e aumento da gordura corporal torna o corpo mais vulnerável a doenças crônicas e riscos relacionados a saúde como as quedas consequentemente as fraturas. A fratura de fêmur está entre as mais frequentes na população idosa, podendo ser geradas por uma simples queda até mesmo da própria altura levando o idoso a sofrer o trauma. Após sofrer a fratura de fêmur o idoso vivencia uma grande alteração em sua qualidade de vida, pois após esse processo na maioria das vezes passa por um tratamento cirúrgico, o que leva a uma queda na qualidade de vida e independência funcional. Nesse contexto, algumas doenças tendem a surgir decorrentes do longo período de reabilitação, o que gera uma redução drástica na capacidade física e funcional do idoso. A atuação fisioterapêutica, de forma geral, é indispensável na saúde da população idosa, e se torna ainda mais importante após alguma fratura, especificamente a de fêmur, pois garante assim a retomada de sua autonomia o mais precoce possível. Trata-se de uma revisão bibliográfica narrativa na qual os artigos foram acessados por meio das bases de dados publicados entre 20II a 202I. De acordo com o estudo conclui-se que a fisioterapia tem uma importante atuação na reabilitação e também na prevenção de complicações cirúrgicas, atuando no pré e pós-operatório das fraturas de fêmur, buscando a funcionalidade do paciente idoso.

Palavras-chave: Fraturas proximal do fêmur. Idosos . Capacidade funcional. Fisioterapia.

ABSTRACT: Aging is a stage of life influenced by several factors and determinants, such as the decrease in physiological functions and physical capacities. The loss of strength, bone mass, flexibility, speed, reduced muscle mass and increased body fat makes the body more vulnerable to chronic diseases and health-related risks such as falls and fractures. Femur fractures are among the most frequent in the elderly population, and can be

\footnotetext{
IAcadêmica do Curso de Fisioterapia na Instituição de Ensino - UNIBRÁS, E-mail: alana.freitası32@outlook.com

${ }^{2}$ Orientadora, Especialista em Fisioterapia e Professora na Instituição de Ensino - UNIBRÁS, Unidade Rio Verde, E-mail: kauaravvieira@gmail.com
} 
generated by a simple fall even from their own height, leading the elderly to suffer trauma. After suffering a femur fracture, the elderly experience a major change in their quality of life, as after this process they most often undergo surgical treatment, which leads to a drop in quality of life and functional independence. In this context, some diseases tend to arise from the long period of rehabilitation, which generates a drastic reduction in the physical and functional capacity of the elderly. Physiotherapeutic action, in general, is essential in the health of the elderly population, and becomes even more important after a fracture, specifically that of the femur, as it guarantees the resumption of their autonomy as soon as possible. This is a narrative bibliographic review in which the articles were accessed through databases published between 20II and 2021. According to the study, it is concluded that physiotherapy has an important role in rehabilitation and also in the prevention of surgical complications, acting in the pre- and postoperative period of femur fractures, seeking the functionality of the elderly patient.

Keywords: Proximal femur fractures. Elderly. Functional Capacity. Physiotherapy.

\section{INTRODUÇÃO}

A fratura do fêmur está entre as lesões traumáticas mais comuns na população idosa, uma vez que, o osso apresenta a capacidade de transmitir a carga durante o movimento. Essa lesão é considerada um grande problema de saúde pública, devido à morbimortalidade, longos tempos de internação e problema social para quem acompanha tais doentes (SANTOS et al., 2021).

Os traços do envelhecimento se alteram dependendo de cada indivíduo, isso ocorre mesmo que estes sejam expostos aos mesmos determinantes ambientais. Tais características podem apresentar-se internas ou externamente como: perda da massa muscular, articulações enrijecidas, diminuição da estatura, ombros arredondados, desgaste das vértebras, metabolismo mais lento, equilíbrio precário, visão e audição menos aguçada e fraturas ósseas (BORTOLON et al., 20II). Dentre as características mencionadas, vale ressaltar que as fraturas constituem um grupo de consequências externas diretamente ligadas ao envelhecimento e está associada à diminuição significativa da capacidade funcional do idoso (SOARES et al., 2014).

A capacidade funcional é um preditor importante na qualidade de vida da pessoa idosa, e pode ser dividida em dois domínios, que compreendem as atividades físicas de vida diária (AFVD), caracterizadas por ações executadas com a finalidade de autocuidado 
Revista Ibero- Americana de Humanidades, Ciências e Educação- REASE

para a manutenção da sobrevivência como alimentar-se, banhar-se, vestir-se e arrumar-se e,

atividades instrumentais da vida diária (AIVDs), definidas por ações mais complexas que promovem interação do indivíduo com o ambiente e com a sociedade, as quais englobam a avaliação da capacidade do idoso de como usar um telefone, fazer compras, preparar alimentos, arrumar a casa, lavar roupas, pegar ônibus, ingerir remédios e manusear as próprias finanças (RAMOS et al., 2013).

As quedas estão relacionadas por uma perda do equilíbrio estático ou dinâmico, que também podem ser associadas a problemas do sistema osteoarticular sendo patologias adquiridas como a osteoporose, neurológico sendo patologias que afetam a região motora/ sensitiva, ou até mesmo pela perda da funcionalidade (CARVALHO; BOCCHI, 2017).

Nos dias atuais, é crescente a consciência de que as fraturas afetam substancialmente a qualidade de vida dos pacientes idosos e de que as mesmas configuram um expressivo problema de saúde pública, visto que, estão associadas a altos custos econômicos, problemas sociais e aos índices crescentes de morbimortalidade. Pacientes com fraturas, por vezes, tornam-se impossibilitados de trabalhar, limitados em sua inserção social, atividades recreativas e, emocionalmente angustiados, portanto, requerem substancial cuidados médicos, psicológicos e assistência nas atividades da vida diária (OLIVEIRA et al., 20II).

O estudo tem por objetivo de fazer uma revisão da literatura sobre a eficácia da fisioterapia na reabilitação de pacientes idosos após se submeterem a um procedimento cirúrgico de reconstrução da fratura proximal de fêmur, garantindo assim uma melhor capacidade funcional aos mesmos.

\section{MATERIAIS E MÉTODOS}

Este artigo trata-se de uma revisão narrativa da literatura que foi realizada por meio de uma pesquisa exploratória, bibliográfica e descritiva, de artigos, revistas cientificas, livros e teses de mestrados e doutorados que citam abordagem fisioterapêutica no paciente idoso com fratura de fêmur. 
Revista Ibero- Americana de Humanidades, Ciências e Educação- REASE

O levantamento dos artigos científicos foi realizado durante 2011 a 2021, por meio das seguintes base de dados: Scientific Eletronic Library Online (Scielo), Google Acadêmico, Biblioteca Virtual em Saúde (BVS), revistas científicas, livros, dissertações de mestrado e

teses de doutorado, utilizando como critério de busca os descritores: fisioterapia, fraturas do fêmur, idoso, fratura proximal do fêmur e reabilitação.

Os dados foram selecionados por meio de uma leitura exploratória do material utilizado, com leituras mais aprofundadas, com objetivo de ordenar e sumariar informações contidas, a fim de elaborar e apresentar os resultados do estudo.

\section{REFERENCIAL TEÓRICO}

\section{Definição e fisiopatologia}

As fraturas ósseas são o resultado de uma sobrecarga única ou múltipla, com uma magnitude que excede o limite suportado pelo osso, ocorrendo numa fração de milissegundos. O efeito mecânico de uma fratura consiste primariamente numa perda da continuidade óssea, levando a uma mobilidade patológica, perda da função de suporte ósseo e ao aparecimento de dor (FERNANDES et al., 2013).

A taxa de crescimento da população idosa vem aumentando consideravelmente e dados do ministério da saúde (MS), revelam que em 2025 o Brasil será o sexto país no mundo com maior número de pessoas idosas (FERREIRA et al., 2013). Com isso tem-se a preocupação com os cuidados e bem estar com essas pessoas que tem uma maior vulnerabilidade de sofrer uma queda que podem resultar em algum tipo de fratura, onde estas ocorrem na região proximal do fêmur deixando o mesmo imobilizado por um longo período, Algumas alterações podem estar relacionadas ao processo de envelhecimento, podendo eles ser associados a fatores que podem ter ligação com o uso de medicamentos, fatores domiciliares, baixa acuidade visual, fatores externos, como calçadas irregulares, iluminação inadequadas, dentre outros (SILVA et al., 20II).

Nota-se um aumento muito significativo na incidência das fraturas de extremidades proximal do fêmur nos últimos anos, devido especialmente ao aumento da 
Revista Ibero- Americana de Humanidades, Ciências e Educação- REASE

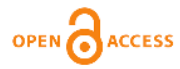

população idosa. As fraturas da região do colo do fêmur são representadas por $84 \%$ das lesões ósseas em pessoas acima de 60 anos, sendo considerado um grande problema de saúde pública, pois estão relacionadas ao aumento da taxa de mortalidade, gastos hospitalares, de incapacidade funcional e problemas sócio familiares na população idosa (GUERRA et al., 2016).

As fraturas da extremidade proximal do fêmur são consideradas todas as fraturas referentes ao segmento ósseo demarcado proximalmente pelo limite inferior da cabeça do fêmur e distalmente por um nível situado cinco centímetros abaixo do limite inferior do pequeno trocanter (BARREIRA, 2015). Tal fratura, transcorre geralmente por traumas de baixa energia, como quedas que podem estar associadas a fraqueza ou sensação de tontura, relacionadas a diversos fatores, dentre eles: idade avançada, osteoporose, diminuição da força muscular, geometria do quadril, pouca ingestão de cálcio e vitamina $\mathrm{D}$ e predisposição genética (ESQUENAZI et al., 2014).

Esse tipo de fratura representa $84 \%$ das lesões ósseas encontradas em pessoas acima dos 60 anos, constitui-se um problema de saúde pública e causa importante de mortalidade, incapacidade funcional, gastos médico-hospitalares excessivos e problemas sócio familiares nessa população (GUERRA et al., 2017). A incidência das fraturas do fêmur proximal já aumentou de forma importante nos últimos anos e deverá duplicar nos próximos 25 anos devido ao já citado aumento da expectativa de vida da população (PETROS et al., 2017).

O risco da ocorrência de fratura aumenta significativamente com o avançar da idade. A previsão é de que $12 \%$ dos idosos que vivem na comunidade sofram algum tipo de fratura durante a vida e, esta previsão aumenta para 30\% da possibilidade de um episódio de fratura em idoso que se encontra institucionalizado. Após fratura, cerca de $5 \%$ dos idosos são hospitalizados devido a ocorrência de fratura grave, principalmente por fratura de quadril e fratura do colo do fêmur. Onde, um em cada três idosos que sofreram uma fratura grave, um destes pacientes vira a óbito no prazo de um ano (LOURES et al., 2015).

\section{Anatomia e biomecânica da articulação coxofemoral}

A articulação coxofemoral é constituída por uma parte chamada de cabeça femoral, situada no osso da coxa, e uma cavidade denominada como acetábulo, onde temos os ossos da bacia. A união destas estruturas facilita uma grande estabilidade articular que possui a 
Revista Ibero- Americana de Humanidades, Ciências e Educação- REASE

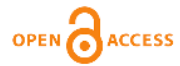

função de sustentar o peso do corpo. A cartilagem que recobre tais estruturas é imensamente importante no papel de impossibilitar o contato ósseo e facilitar o deslizamento entre as articulações. (SILVA, 2012)

O fêmur possui uma cabeça esférica que se encaixa em um soquete profundo chamado acetábulo situado na pelve, revestido por uma massa de tecido adiposo, cápsula e

ligamentos (PRENTICE, 2012). A cabeça do fêmur com sua forma convexa, durante o movimento da coxa desliza no sentido oposto da mesma (LIPPERT, 2013).

Entre o osso do quadril e o fêmur, existe uma estrutura que aumenta a estabilidade articular, a mesma é chamada de lábio (ou lábrum) - borda acetabular revestida de fibrocartilagem - tal estrutura aprofunda o acetábulo permitindo a estabilidade articular entre o osso do quadril e o fêmur causando uma pressão intra-articular negativa na articulação do quadril. (ALMEIDA, 2017). Por suportar muita carga, o quadril acaba sofrendo danos irreversíveis que comprometem a articulação, causando limitações nas funções e prejudicando o desenvolvimento de AVDs, com isso a coxofemoral é uma das articulações mais afetadas por lesões de natureza múltipla (HOUGLUM; BERTOTI, 2014).

Segundo Lippert (2013), a articulação contém três planos de movimento: o plano sagital: com aproximadamente $120^{\circ}$ de flexão e $14^{\circ}$ de hiperextensão; o plano frontal: com abdução aproximada de $45^{\circ}$; o plano horizontal (transversal): as rotações mediais (rotação interna) e laterais (rotação externa) é possível obter aproximadamente $45^{\circ}$ de rotação em cada direção a partir da posição anatômica.

A articulação do quadril é cercada por uma cápsula articular forte composta por quatro ligamentos, o mais importante é o iliofemoral, um feixe bastante resistente, situado anteriormente à articulação, está intimamente unido à cápsula e serve para reforçá-la. Estes tecidos mantêm o quadril em movimento para uma posição extrema, que poderia deslocar a articulação (ALBINO et al., 2012). A articulação da região do quadril realiza os movimentos de flexão, extensão, abdução, adução, rotação medial e rotação lateral. A articulação da pelve realiza anteroversão, retroversão, inclinações laterais e rotação pélvica (TORTORA; NIELSEN, 2013). 
Revista Ibero- Americana de Humanidades, Ciências e Educação- REASE

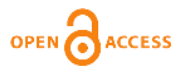

\section{Alterações funcionais decorrentes da fratura}

As consequências das fraturas de fêmur proximal nos idosos podem ser devastadoras, alterando o equilíbrio físico, mental, funcional e social. Além disso, acarreta danos familiares, uma vez que é criada uma dependência por perda de autonomia após o trauma, gerando problemas internos. A fragilidade e o equilíbrio são itens mensuráveis que tendem a drasticamente afetarem a vida do idoso, principalmente nos casos após o trauma, onde

pode haver novamente o risco de queda, o aumento de hospitalizações e o risco de morte (FILHO et al., 2019; SILVA et al., 2020).

Carvalho et al. (2017), relatam sobre os efeitos negativos mais comuns após o idoso sofre uma fratura de fêmur, entre eles estão a tristeza e a insegurança em virtude da dor, do estado de dependência e da incerteza do alcance da reabilitação plena. As dificuldades enfrentadas pelos idosos referem-se à restrição de atividades, imobilidade, prejuízos psicológicos, medo de sofrer novas quedas e de risco de morte, além dos prejuízos relacionados à família, a custos financeiros e a disponibilidade para o cuidado. A consequência é uma série de aspectos negativos em seu cotidiano, pois o idoso fica impedido de desfrutar de uma vida independente e ativa.

Há, ainda, perda progressiva da capacidade de as articulações absorverem pressões, tornando-se mais rígidas e as cartilagens menos elásticas; declínio do poder muscular, levando à desaceleração dos movimentos e à perda da coordenação; aparecimento de dificuldades com o equilíbrio, destacando-se, como fatores causadores, a perda da propriocepção dos tecidos nas superfícies de apoio com carga e a fraqueza (BENTO et al., 20II).

Além disso, a incapacidade física total ou parcial após a fratura é outro grande problema, sendo que 50\% dos pacientes tornam-se restritos ao leito ou à cadeira de rodas e, daqueles que conseguem regressar ao domicílio, 25 a 35\% passam a necessitar de cuidadores ou de algum dispositivo que os auxiliem na locomoção (SOARES et al., 2014).

\section{Capacidade funcional vs. qualidade de vida}


Revista Ibero- Americana de Humanidades, Ciências e Educação- REASE

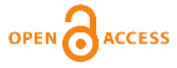

O processo de envelhecimento é marcado pela diminuição progressiva e fisiológica da capacidade funcional dos idosos (senescência), que pode evoluir para sobrecarga e estresse, culminando na perda significativa da habilidade funcional (senilidade) e ao comprometimento de capacidades mentais e físicas, acarretando, assim, a dependência no desenvolvimento das atividades de vida diária (SOARES et al., 2014).

A fragilidade da idade traz diversas alterações nas estruturas corporais alterando a forma como deveriam ser trabalhadas: fraqueza muscular, fragilidade óssea, índice de massa

corporal baixo, tento como as principais a instabilidade e a incapacidade física que são significativamente diminuídas (RUNDH; GOTTFRIDSSON, 2015).

O declínio da capacidade funcional é uma das características mais marcantes do processo de envelhecimento e envolve perda de força, equilíbrio, flexibilidade, agilidade e coordenação motora, que constituem variáveis afetadas diretamente por alterações neurológicas e musculares, dificultando a realização das atividades diárias e a manutenção de um estilo de vida saudável (SANTOS et al., 2013).

A capacidade funcional é um preditor importante na qualidade de vida da pessoa idosa, e pode ser dividida em dois domínios, que compreendem as atividades físicas de vida diária (AFVD), caracterizadas por ações executadas com a finalidade de autocuidado para a manutenção da sobrevivência como alimentar-se, banhar-se, vestir-se e arrumar-se e, atividades instrumentais da vida diária (AIVDs), definidas por ações mais complexas que promovem interação do indivíduo com o ambiente e com a sociedade, as quais englobam a avaliação da capacidade do idoso de como usar um telefone, fazer compras, preparar alimentos, arrumar a casa, lavar roupas, pegar ônibus, ingerir remédios e manusear as próprias finanças (RAMOS et al., 2013).

As quedas estão diretamente associadas à perda da capacidade funcional nos pacientes idosos, seja como consequência, seja como fator causal, pois podem, por si só, levar à perda da funcionalidade, que pode também tornar-se um fator de risco para novas quedas e, assim, trazer mais prejuízos e maior dependência (SANTANA et al., 2015). 
Revista Ibero- Americana de Humanidades, Ciências e Educação- REASE

Dentre as características mencionadas, vale ressaltar que as fraturas constituem um grupo de consequências externas diretamente ligadas ao envelhecimento e está associada à diminuição significativa da capacidade funcional do idoso. Sua ocorrência tende a aumentar com a idade, chegando a $51 \%$ nos indivíduos com mais de 85 anos. Em geral, as fraturas se devem à marcha instável e perda do equilíbrio, decorrente da insuficiência súbita dos mecanismos neuro-sensoriais e osteoarticulares envolvidos na manutenção da postura. As principais consequências das fraturas são o aumento do risco de morte, restrição de atividades, declínio da saúde global e o aumento do risco de institucionalização (SOARES et al., 2014).

\section{Tratamento pós-fratura proximal de fêmur}

A escolha do tratamento definitivo depende do estado geral do paciente e do padrão da fratura. Diante dos diagnósticos apresentados pelo paciente segue-se uma linha de tratamento que pode ser cirúrgico ou conservador. Dentro da classe de tratamento para pacientes portadores desse tipo de trauma, o mais indicado é o cirúrgico. $O$ tipo de cirurgia vai depender do local atingido, da configuração da fratura e do nível de atividade do paciente. As taxas de complicações e mortes são menores quando a fratura é fixada internamente, permitindo a mobilização precoce (SANTOS et al., 202I).

O local da fratura (colo do fêmur) determina o tipo de cirurgia, e é preciso considerar ainda se há ou não desvio do osso e qual o nível de atividade do paciente. Podem ser usados, como recursos cirúrgicos, parafusos e placas, hastes intramedulares, fixação percutânea com fios e parafusos ou prótese de quadril (artroplastia), mais comuns para idosos (KHAN et al., 2009; LIBRERO et al., 2012; RICCI et al., 2012).

A fratura de colo de fêmur não desviada tem indicação de tratamento cirúrgico de fixação, geralmente consistindo em osteossíntese com aplicação de implantes extramedulares, o que se mostra mais benéfico do que o tratamento conservador, ou não cirúrgico. A idades cronológicas e/ou fisiológicas menores são os principais fatores para que se escolha esse tipo de tratamento. Já na fratura de colo de fêmur desviada, o tratamento indicado é um procedimento cirúrgico de substituição, podendo ser artroplastia parcial ou total, dependendo das características individuais do paciente (BRASIL, 2018; GIORDANO et al., 2019). 
Revista Ibero- Americana de Humanidades, Ciências e Educação- REASE

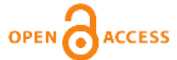

Os principais métodos cirúrgicos utilizados são a osteossíntese e a artroplastia (PIRES et al., 2012). A osteossíntese é um "procedimento cirúrgico que visa restaurar a continuidade anatômica dos ossos” (BRASIL, 2013), podendo-se utilizar pinos metálicos, parafusos, placas, entre outros dispositivos dessa espécie, sendo assim uma cirurgia de fixação (BRASIL, 2013). Já a artroplastia é "um procedimento cirúrgico que visa substituir parcial ou totalmente uma articulação, com a finalidade de restaurar a mobilidade”, sendo assim uma cirurgia de substituição (BRASIL, 2013).

As fraturas de estágio I e II de Garden são tratadas com vários parafusos inseridos. Já nos estágios III e IV, o tratamento é realizado com a substituição da cabeça e do colo do fêmur, a hemiartroplastia que permite uma mobilização pós-cirúrgica parcialmente rápida e anulação da necessidade de uma segunda cirurgia (GUIMARÃES et al., 20II).

\section{Atuação fisioterapêutica no pós-operatório de fraturas do fêmur}

Segundo Carneiro et al. (2013), o objetivo da fisioterapia no tratamento pósoperatório de pacientes com fratura do fêmur é aumentar a força muscular, melhorar a segurança e eficiência da deambulação, fornecendo assim, maior independência ao paciente. Mas para um início seguro da fisioterapia é de extrema importância que o profissional conheça o tipo de fratura, assim como o material usado para fixação cirúrgica, pois esses dados irão interferir na conduta do tratamento, que inclui o tempo de deambulação, a descarga de peso no membro, bem como restrições em alguns movimentos. A importância da fisioterapia durante o período hospitalar tem como seu principal objetivo promover a reabilitação e recuperação no pré e pós-operatório, estimulando o seu retorno às atividades, e melhorando o seu bem-estar e a sua qualidade de vida (CARNEIRO et al., 2013).

A fisioterapia durante o período hospitalar tem o objetivo de promover orientações quanto ao pós-operatório e estimular o retorno às atividades de vida diária, desta forma melhorando a qualidade de vida do paciente. As condutas realizadas durante a internação variam conforme a necessidade do paciente, com destaque para mobilizações passivas, exercícios ativo-assistidos e ativos, exercícios resistidos, exercícios metabólicos, técnicas 
respiratórias de reexpansão e desobstrução, transferências e tomadas de peso, treino de equilíbrio e prescrição de muletas (CARNEIRO et al., 2013).

Bento et al. (2011), ressaltam que, deve-se iniciar o tratamento ainda no período hospitalar promovendo orientações ao paciente no pós-operatório com intuito de buscar preservação da função corporal para evitar que ocorra úlceras de decúbito ou deformidades. Durante a internação realizam-se as condutas de acordo com a necessidade do paciente, utilizando mobilizações passivas, exercício ativos, ativo-assistido e resistidos, técnicas respiratórias, treino de equilíbrio e prescrição de muletas e ou andadores para auxiliar na deambulação.

A estimulação elétrica nervosa transcutânea (TENS) é uma das técnicas fisioterapêuticas bastante utilizadas para diminuir a dor aguda ou crônica e atua substituindo ou diminuindo os analgésicos, e assim, faz com que o paciente se movimente mais rápido no leito (ALVES et al., 2015). A Corrente Russa também tem mostrado resultados excelentes no tratamento de diversas doenças, trabalhando o ganho de força muscular causada pela imobilização e contribuindo de forma positiva para a reabilitação do paciente (ALVES et al., 2017).

É essencial o início da amplitude de movimento pós-operatória imediata do joelho para evitar rigidez e perda de função. Isso é alcançado com fisioterapia precoce e fortalecimento dos membros inferiores. Uma joelheira articulada pode ser usada para proteger contra tensões em varo e valgo na fratura, se houver preocupação com a qualidade da fixação da fratura (VON KEUDELL et al., 2016).

Arndt et al. (20II), demonstraram a importância de serem realizados movimentos passivos progredindo para exercícios ativos de flexão extensão do joelho, quadril e tornozelo sem carga total no membro nos primeiros dias de pós-operatório. A mobilização precoce do paciente é prioritária e constitui um importante variável no processo de recuperação, entretanto, se o paciente não puder deambular, realizam- se os exercícios ativos de acordo com o limite funcional e passivos ainda no leito para que possa progredir futuramente as atividades em cadeira de rodas. 
Revista Ibero- Americana de Humanidades, Ciências e Educação- REASE

Exercícios respiratórios, com técnicas de desobstrução das vias aéreas e a drenagem postural, entre outras manobras respiratórias, tem a intenção de normalizar o fluxo respiratório. A elevação das extremidades dos membros inferiores auxilia o retorno venoso diminuindo o edema, pois o paciente passa longo período restrito ao leito na fase hospitalar (VASCONCELOS, 2012).

Considera-se que a massagem terapêutica tenha estimulado os receptores periféricos, provendo relaxamento e mobilização muscular, e melhora no retorno venoso e linfático. Atribuiu-se ao ultrassom, a melhora do quadro álgico, a diminuição da rigidez articular, o aumento do fluxo sanguíneo e a redução dos espasmos musculares, enquanto a cinesioterapia, a eletroterapia e a hidroterapia auxiliaram no fortalecimento muscular,

reeducação do movimento e melhora da circulação sanguínea (VICENTE; HUMMEL, 2019).

A cinesioterapia utiliza-se de todas as técnicas reabilitadoras baseadas no movimento. As metas de tratamento pelo exercício são: minimizar os efeitos da inatividade, corrigir a ineficiência de músculos específicos ou grupos musculares, reconquistar a amplitude normal de movimento da articulação e encorajar o paciente a usar a habilidade reconquistada no desempenho de atividades funcionais, acelerando a reabilitação. São aplicadas como técnicas cinesioterapêuticas exercícios com movimentos passivos, ativo- assistidos, ativo-livres e ativoresistidos (NASCIMENTO et al., 2013).

A hidroterapia é um dos recursos mais antigos da fisioterapia, que utiliza propriedades físicas da água em conjunto com a cinesioterapia com propósitos terapêuticos, sendo considerada segura e eficaz para a reabilitação pós-cirúrgica, pois minimiza o estresse biomecânico nos músculos e articulações, atuando assim nas desordens musculoesqueléticas e na melhora do equilíbrio. Assim o meio aquático proporciona aumento da força muscular e da amplitude articular, relaxamento muscular, diminuição temporária do nível de dor, melhora da confiança e da capacidade funcional proporcionando qualidade de vida ao paciente (ANDRIOLLO-BASSO e ROSA, 2012).

Silva et al. (201I) concluíram em seu estudo que a fisioterapia realizada através de exercícios de alongamento e de treinamento do equilíbrio e da força trouxeram benefícios 
Revista Ibero- Americana de Humanidades, Ciências e Educação- REASE

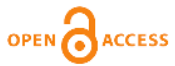

tanto ao equilíbrio quanto à marcha. Para Carneiro et al. (2013), há uma tendência de que os exercícios de fortalecimento seriam a chave para permitir a melhora funcional dos pacientes fraturados.

Nunes e Mejia (2012), afirma que o tratamento fisioterapêutico do pós-operatório se mostrou essencial, permitindo aumentar a amplitude de movimento e a força muscular, por meio de um programa de exercícios de alongamento e fortalecimento, além de permitir deambulação precoce com auxílio de muletas e carga parcial do membro operado, restaurando assim o padrão da marcha ao final da fisioterapia e garantindo que o paciente tenha uma recuperação eficaz e retorne de imediato as atividades de vida diária sem qualquer sinal de sequela ou auxílio para se locomover.

Bogni (2013), concluiu que os exercícios de resistência melhoram a massa muscular e massa óssea, pois o osso se adapta a carga mecânica proporcionada pelo exercício através do efeito piezoelétrico, estimulando assim a ação sobre os osteoblastos para formar osso novo. Os ossos, assim como os músculos, tendem a se tornar mais fortes e resistentes quanto mais forem usados e exercitados, com certos limites. Assim o exercício de resistência demonstrar ser eficaz para a aceleração da calcificação óssea da fratura e melhorar da força muscular.

Os exercícios que englobam o ganho de amplitude de movimento, força muscular e treino funcional, assim como o treino de equilíbrio, proprioceptivo e de postura, são incluídos em uma fase do tratamento na qual a consolidação óssea seja satisfatória, e que permita uma descarga de peso total ou parcial no membro afetado (BENTO et al. 2orI). Candeloro e Silva (2012), descrevem que algumas atividades devem ser executadas dentro do limite e intensamente do idoso tolerado. Essas atividades envolvem exercícios que aumentem a mobilidade, a força, equilíbrio, o treino de marcha, a evolução da aferência sensorial e a facilidade em transferência.

Com a evolução do tratamento é necessário que o paciente realize exercícios de propriocepção, estes influenciam na conciliação do senso de posição articular e neuromuscular, trabalhando equilíbrio dinâmico e movimentos alternados, mantendo a estabilidade dos movimentos e melhorando o desempenho do controle postural. É 
Revista Ibero- Americana de Humanidades, Ciências e Educação- REASE

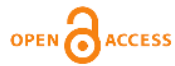

necessário ainda reaprender a deambular, o qual se deve realizar transferência de peso adequado durante o ciclo da marcha coordenando assim seu equilíbrio estático e dinâmico para manter a estabilidade postural, pois o membro fraturado gera uma condição de alteração de equilíbrio estático e sobrecarga no membro saudável que precisa ser restabelecida, respeitando o condicionamento físico dos mesmos (ALENCAR et al., 2012).

\section{CONCLUSÃO}

A fratura do fêmur proximal, acomete mais paciente idoso, isso acontece devido a maior tendência a acidentes doméstico nesta fase de vida: como quedas de própria altura, diminuição da acuidade visual, diminuição de força muscular e reflexos (reação de defesa) doenças neurológicas, e a facilidade de fraturas pela presença de osteoporose, dentre outras causas.

As quedas estão diretamente associadas a perda da capacidade funcional, sendo cada vez mais frequentes entre os idosos. As quedas produzem complicações que alteram negativamente a qualidade de vida, afetando tanto fisicamente como psicologicamente, levando muitos a paralisarem de suas atividades rotineiras e também a manter o isolamento social.

A atuação fisioterapêutica, de forma geral, é indispensável na saúde da população idosa, podendo atuar tanto na prevenção de quedas, como a elaboração de programas preventivos, quanto no pré-operatório e pós-operatório imediato, visando uma melhora da qualidade de vida.

Como exercícios de fortalecimento, de equilíbrio e de marcha, ambos trabalhados em sessões de fisioterapia, as quais levam o aumento da força muscular; melhora o equilíbrio, a flexibilidade, a coordenação motora e a propriocepção, além do ensinamento de exercícios que futuramente poderão ser realizados sozinhos pelo paciente, tornando assim uma vida saudável e prazerosa. Diante desse contexto a atuação do fisioterapeuta é extremante importante.

\section{REFERÊNCIAS}


I. ALBINO, I.LR.; FREITAS, C.R.; TEIXEIRA, A.R.; GONÇALVES, A.K.; SANTOS, A.M.P.V.; BÓS, A.J.G. Influência do treinamento de força muscular e de flexibilidade articular sobre o equilíbrio corporal em idosas. Revista Brasileira de Geriatria e Gerontologia. v. 15, n. I, p. 17-25, 2012.

2. ALENCAR, A.G.M.; ISACKSOON, R.R.A.; MEIRELES, K.A.D.; CAMPOS, N.G. Abordagem terapêutica em paciente pós-fratura de tornozelo e compressão da coluna lombar: relato de caso. Revista Fisioterapia e Saúde Funcional. v. I, n. 2, p. 6r-65, 2012.

3. ALMEIDA, T.I.S. Fisioterapia pós-artroplastia total de quadril em adultos acometidos por displasia do desenvolvimento do quadril: revisão de literatura. 45f. Monografia (Graduação em Fisioterapia). Faculdade de Educação e Meio Ambiente Ariquemes, 2017.

4. AMARANTE, C.F.S.; CARDOSO, D.B.; ANDRADE, F.J.S.; PERDIGAO, K.M.; LEMOS, L.V.M.; RODRIGUES, M.; LIMA-JÚNIOR, M.R.; GOMES, S.A.;

CARVALHO- JÚNIOR, L.H. Fraturas do fêmur proximal em idosos, Revista Médica de Minas Gerais, v. 2I, n. 2, p. 21-24, 2011.

5. ALVES, E.M.S.; RABÊLO, T.N.; SANTOS, M.G.R.; SOUZA, I.G.; LIMA, P.A.L.; SANTANA, L.S. Eletroestimulação nervosa transcutânea para analgesia pós-operatória em cesariana. Revista Dor. v. 4, n. 16, p. 264, 2015;

6. AlVES, D.S.; PRADO, R.P.; BRAZ, A.G.; MACHADO, E.C.F.; MORAES, F.A. Análise comparativa do pico de força de controle motor do músculo tibial anterior após cinesioterapia e estimulação neuromuscular. Revista Brasileira de Ciência e Movimento. v. 25, n. 4, p.50-5I, 2017.

7. ANDRADE, J.P.; SILVA, D.Z.; PATRÍCIO, D.S.; Incidência de casos de fratura de fêmur no Brasil entre os anos de 2015 e 2020 através de dados epidemiológicos do datasus: faixa etária e gênero. Scientia Generalis, v. I, n. 3, p. 84-91, 2020.

8. ANDRIOLLO-BASSO, D.B.; ROSA, T.S.M. Fisioterapia aquática na prevenção de quedas para a terceira idade: considerações teóricas. Universidade Federal de Santa Maria, 2012. 
9. ARNDT, A.B.M.; TELlES, J.L.; KOWALSKI, S.C.; O custo direto da fratura de fêmur por quedas em pessoas idosas: análise no setor privado de saúde da cidade de Brasília, 2009. Revista Brasileira de Geriatria e Gerontologia. v. I4, n. 2, p. 221-231, 2011.

Io. BARREIRA, E.M.G. Idosos com fratura proximal do fémur: análise das quedas e alterações funcionais. I2ıp. Dissertação de Mestrado em Enfermagem de Reabilitação, Escola Superior de Saúde de Bragança - Instituto Politécnico de Bragança, Bragança Portugal, julho de 2015 .

II. BENTO, N.T.; VIDMAR, M.F.; SILVEIRA, M.M.; WIBELINGER, L.M. Intervenções fisioterapêuticas no pós-operatório de fratura de fêmur em idosos. Revista Brasileira de Ciências da Saúde, ano. 9, n. 27, p. 42-48, 2011.

12. BOGNI, F.H. Efeito do envelhecimento e do treinamento resistido sobre os ossos de ratos. Dissertação (Mestrado em Ciências Fisiológicas). Universidade Federal de São Carlos, 2013.

13. BORTOLON, P.C.; ANDRADE, C.L.T.; ANDRADE, C.F. O perfil das internações do SUS para fratura osteoporótica de fêmur em idosos no Brasil: uma descrição do triênio 2006-2008. Caderno de Saúde Pública, v. 27, n. 4, p. 733-742, 2011.

14. BRASIL, Ministério da Saúde. Conselho Nacional de Saúde. Resolução № 466 de I2 de dezembro de 2012. Diário Oficial da União, 2012.

15. BRASIL, Ministério da Saúde. Secretaria executiva. Secretaria de Atenção à Saúde. Glossário temático: traumatologia e ortopedia. zed. Brasília: Ministério da Saúde, 2013.

16. BRASIL, Secretaria de Atenção à Saúde e Secretaria de Ciência, Tecnologia e Insumos Estratégicos. Portaria conjunta no 2 I de 24 de setembro de 2018. Diretrizes Brasileiras para o Tratamento de Fratura de Colo de Fêmur em Idosos, 2018.

17. CANDELORO, J.M.; SILVA, R.R. Proposta de protocolo hidroterapêutico para fraturas de fêmur na terceira idade. Trabalho de Conclusão de Curso (Pós-graduação em Hidroterapia). Universidade Bandeirante de São Paulo, 2012. 
Revista Ibero- Americana de Humanidades, Ciências e Educação- REASE

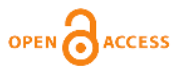

I8. CARNEIRO, B.B.; ALVES, D.P.L.; MERCADANTE, M.T. Fisioterapia no pósoperatório de fratura proximal do fêmur em idosos: revisão da literatura. Acta Ortopédica Brasileira, v.21, n. 3, p. 175-178, 2013.

19. CARVALHO, C.J.A.; BOCCHI, S.C.M. Idoso reconhecendo-se vulnerável a quedas na concretude da fratura do fêmur. Revista Brasileira de Enfermagem, v. 70, n. 2, p. 296303, 2017.

2o. ESQUENAZI, D.; SILVA, S.R.B.; GUIMARÃES, M.A.M. Aspectos fisiopatológicos do envelhecimento humano e quedas em idosos. Revista HUPE, v. 13, n. 2, p. 11-20, 2014.

2r. FERNANDES, M.G.A.; ROCHA, D.N.; SILVA, J.V.L.; SILVA, J.V.L.; FONSECA, E.M.M.; BARBOSA, M.P. Análise biomecânica das fraturas do fémur: estudo preliminar de uma haste intramedular bloqueada. In: $5^{\circ}$ Congresso Nacional de Biomecânica, Espinho, Portugal. Actas do 5을 Congresso Nacional de Biomecânica, p. 313-317, 2013.

22. FERREIRA, A.C.; ALMEIDA, D.R.; CAMPOS, W.N.L.; CAMPOS, F.M.C.; TOMAZELLI, R.; ROMÃO, D.F. Incidência e caracterização de idosos na clínica

ortopédica do Hospital Regional Cáceres por fratura de fêmur. Revista Eletrônica de Gestão em Saúde. v. 4, n. 2, p. 1932-1941, 2013.

23. FILHO, J.A.; SILVA, A.D.C.; MENDES-JÚNIOR, A.F.; PEREIRA, F.J.C.; OPPE, I.G.; LOURES, E.A. Predictive factors of death after surgery for treatment of proximal femoral fracture. Revista Brasileira de Ortopedia. v. 54, n. 4, p. 402-407, 2019.

24. GIORDANO, V.; GIORDANO, M.; AQUINO, R.; GROSSI, J.O.; SENNA, H.; $\mathrm{KOCH}, \mathrm{H} . \mathrm{A}$. Como os cirurgiões ortopédicos tratam a fratura desviada do colo do fêmur no paciente de meia idade? Pesquisa brasileira com 78 cirurgiões ortopédicos. Revista Brasileira de Ortopedia. v. 54, n. 3, p. 288-294, 2019.

25. GUERRA, H.S.; SOUSA, R.A.; BERNARDES, D.C.F.; SANTANA, J.A.; BARREIRA, L.M. Prevalência de quedas em idosos na comunidade. Revista Saúde e Pesquisa, v. 9, n. 3, p. 547-555, 2016. 
26. GUERRA, M.T.E.; VIANA, R.D.; FEIL, L.; FERON, E.T.; MABONI, J.; VARGAS, A.S. Mortalidade em um ano de pacientes idosos com fratura do quadril tratados cirurgicamente num hospital do Sul do Brasil. Revista Brasileira de Ortopedia. v. 52, n.I, p.17-23, 2017 .

27. HOUGluM, P.A.; BERTOTI, D.B. Cinesiologia Clinica de Bronnstron. 6 ed. Barueri - SP: Manole, 20I4.

28. KHAN, S.K.; KALRA, S.; KHANA, A.; THIRUVENGADA, M.M. PARKER, M.J. Timing of surgery for hip fractures: a systematic review of 52 published studies involving 29I,413 patients. Journal Injury, v. 40, n. 7, p. 692-697, 2009.

29. LIBRERO, J.; PEIRÓ, S.; LEUTSCHER, E.; MERLO, J.; BERNAL-DELGADO, E.; RIDAO, M.; MARTÍNEZ-LIZAGA, N.; SANFÉLIX-GIMENO, G. Timing of surgery for hipfracture and in-hospital mortality: a retrospective population-based cohort study in the Spanish National Health System. BMC Health Services Research, v. I2, n. 15, p. III,2012.4

30. LIPPERT, L.S. Cinesiologia Clínica e Anatomia. 5ed. Rio de Janeiro, 2013.

3r. LOURES, F.B.; CHAOUBAH, A.; MACIEL. V.S.; PAIVA, E.P.; SALGADO, P.P. NETTO, A.C. Custo-efetividade no tratamento cirúrgico das fraturas de quadril em idosos no Brasil. Revista Brasileira de Ortopedia. v.50, n. I, p. 38-42, 2015.

32. MAIA, F.E.S.; GURGEL, F.F.A. Existe fundamento científico para tratamento de fratura de fêmur pela hidroterapia? - uma revisão integrativa. Revista de Atenção à Saúde, v. I4, n. 49, p. 104-110, 2016.

33. MOURÃO, A.L.; VANCONCELLOS, H.A. Geometria do fêmur proximal em ossos de brasileiros. Acta Fisiátrica, v. 8, n. 3, p. I13-119, 2016.

34. NASCIMENTO, M.R.A.; BORDIAK, F.C.; CAMPOS, M.O. Efeito da cinesioterapia na amplitude de movimento de um paciente com osteossíntese em fratura olecraniana. EFDeports Revista Digital, ano 17, n. 177, 2013. 
35. NUNES, A.S.; MEJIA, D.P.M. A eficácia da fisioterapia na reabilitação imediata de paciente pós-operatório de fratura diafisária do fêmur utilizando haste intramedular: revisão bibliográfica, 2012.

36. OLIVEIRA, P.P.; SANDRIN, C.C.; BATISTA, P.H.; MARINHEIRO, L.P.F.; WENDER, M.C.O.; ROISENBERG, F. Relação entre qualidade de vida e fratura vertebral em mulheres idosas residentes no Sul do Brasil. Revista Brasileira de Ginecologia e Obstetrícia, v. 33, n.5, p. 231-237, 2011.

37. PRENTICE, W.E. Fisioterapia na Prática Esportiva. I4ed. Porto Alegre: Artmed, 2012. 38. PETROS, R.S.B.; FERREIRA, P.E.V.; PETROS, R.S.B. Influência das fraturas de fêmur proximal na autonomia e mortalidade dos pacientes idosos submetidos a osteossíntese com haste cefalomedular. Revista Brasileira de Ortopedia. v. 52, p. 57-62, 2017.

39. PIRES, R.E.; PRATA, E.F.; GIBRAM, A.V.; SANTOS, L.E.N.; LOURENÇO, P.R.B.T.; BELLOTI, J.C. Anatomia radiográfica da região proximal do fêmur: correlação com a ocorrência de fraturas. Acta Ortopédica Brasileira. v. 20, n. 2, p. 79-83, 2012.

4o. RAMOS, L.R.; ANDREONI, S.; COELHO-FILHO, J.M.; LIMA-COSTA, M.F.; MATOS, D.L.; REBOUÇAS, M.; VERAS, R. Perguntas mínimas para rastrear

dependência em atividades da vida diária em idosos. Revista Saúde Pública, v. 47, n. 3, p. 506-513, 2013.

4I. RICCI, G.; LONGARAY, M.P.; GONÇALVES, R.Z.; UNGARETTI-NETO, A.S.; MANENTE, M.; BARBOSA, L.B.H. Evaluation of the mortality rate one year after hip fracture and factors relating to diminished survival among elderly people. Revista Brasileira Ortopedia, v. 47, n. 3, p. 304-309, 2012.

42. RUNDH, B.; GOTTFRIDSSON, P. Delivering sports events: the arena concept in sports from a network perspective. Journal of Business \& Industrial Marketing, v. 30, n. 7, p. 785-794, 2015 . 
43. SANTANA, D.F.; REIS, H.F.C.; EZEQUIEL, D.J.S.; FERRAZ, D.D. Perfil funcional, sociodemográfico e epidemiológico de idosos hospitalizados por fratura proximal de fêmur. Revista Kairós Gerontologia, v. I8, n. I, p. 217-234, 2015.

44. SANTOS, E.L.C.; BARBOSA, M.C.; MEDEIROS, J.D.; GRANJA, K.S.B.; CONSTANT, M.H.L.; CALlES, A.C.N. Declínio da capacidade de independência funcional em indivíduos idosos hospitalizados. Ciências Biológicas e da Saúde, v. I, n. 3, p. 91-I00, 2013.

45. SANTOS, L.E.S.; SANTOS, V.V.; NAZIAZENO, S.D.S.; SANTOS, L.S. Fatores causais associados à fratura de fêmur em idosos. Ciências Biológicas e de Saúde - UNIT, v. 6, n. 3, p. 121-134, 2021.

46. SILVA, A.M.; SILVA, R.B.; GUERRA, R.A.; SIQUEIRA, R. Fisioterapia em relação à marcha e ao equilíbrio em idosas. Revista Brasileira de Pesquisa em Saúde. v. 24, n. 3, p. 207-213, 2011.

47. SILVA, F.R. Complicações no pós-operatório de artroplastia total de quadril: assistência e o conhecimento da enfermagem, 2012.

48. SILVA, K.R.; MARTINS, C.I.; SOUZA, F.G.; FARIA, S.M.C.; FERNANDES, M.L.; CAMARGOS, M.C.S. Perfil epidemiológico de pacientes idosos atendidos em um prontosocorro. Revista de Enfermagem UFPE online, 2020.

49. SOARES, D.M.; MELlO, L.M.; SILVA, A.S.; MARTINEZ, E.Z.; NUNES, A.A. Fraturas de fêmur em idosos no Brasil: análise espaço-temporal de 2008-2012. Caderno de Saúde Pública, v. 30, n. 12, p. 2669-2678, 2014.

5o. SOARES, V.T.; RODRIGUES, N.B.; NOWOTNY, J.P.; LIMANA, M.D. Benefícios da microcorrentes no envelhecimento cutâneo. Fisioterapia Brasil, v. 15, n. I, p. 29-34, 2014.

5i. TORTORA, G.J.; NIElSEN, M.T. Princípios da Anatomia Humana. ized. Porto Alegre: Artmed, 2009.

52. VASCONCELOS, L.C. Intervenção fisioterapêutica em paciente com fratura de fêmur: artigo de revisão. Revista Presciência. n. 5, p. 130-I44, 2012. 
53. VICENTE, G.; HUMMEL, J. Tratado de fisioterapia e fisiatria em pequenos animais. São Paulo: Paya, 2019.

54. VON-KEUDELL, A.; SHOJI, K.; NASR, M.; LUCAS, R.; DOLAN, R.; WEAVER, M.J. Treatment options for distal femur fractures. Journal of Orthopaedic Trauma. v. 30, n. 8, p. 25-27, 2016. 